\title{
ON MODELING UTILITY FOR COOPERATIVE SLOTTED ALOHA GAMES
}

\author{
Dandan Wang*, Cristina Comaniciu ${ }^{\dagger}$, Hlaing Minn* and Naofal Al-Dhahir* \\ ${ }^{*}$ Dept. of Electrical Engineering \\ University of Texas at Dallas, Richardson, TX, 75083, USA. \\ Emails:\{dxw053000, hlaing.minn, aldhahir\}@utdallas.edu \\ ${ }^{\dagger}$ Dept. of Electrical Engineering \\ Stevens Institute of Technology, Hoboken, NJ, 07094, USA. \\ Email: \{ccomanic\}@stevens.edu
}

\begin{abstract}
In this paper, we propose a generalized utility function model for cooperative slotted Aloha in a distributed system. Multiuser diversity gains are achieved by appropriately designing the Nash equilibrium thresholds for the selfish users. The network enforces fairness among different users by employing a pricing policy that favors equal access probabilities. This generalized game model provides the flexibility in designing the utility function associated with QoS. Our study on the impact of different utility function models shows that the multiuser diversity gains achieved by different models remain constant under the throughput optimization framework and vary slightly under the revenue optimization framework.
\end{abstract}

Index Terms - Game theory, Aloha, multiuser diversity, pricing, medium access control

\section{INTRODUCTION}

Multiuser diversity has been proposed as an effective technique to maximize the total information-theoretic capacity in the context of multiuser communications [1]. The basic idea of multiuser diversity is to exploit the randomness of fading channels among different users. The larger the dynamic range of channel fluctuations and the number of users, the larger the available multiuser diversity gain is. However, centralized scheduling schemes require significant information exchange between users and the access point to achieve the multiuser diversity gain. Consequently, a distributed access scheme which reduces this information exchange but can still utilize the multiuser diversity is very desirable.

In a distributed system, users may act selfishly to maximize their own performance. In the literature, game-theoretic formulations have become a very useful tool for analyzing medium access protocols, especially in the presence of selfish users that seek to maximize their own performance, and in particular for slotted Aloha [2]. In [3], we proposed a game model associating the channel characteristics with the transmission costs to achieve multiuser diversity gains, in which a Nash equilibrium threshold is designed to account for statistical channel characteristics. However, the game model in [3] assumes that users use the system resources aggressively, which may not be the case for some delay-insensitive services. In this paper, we propose a generalized utility function model and show the

${ }^{1 *}$ The work of these authors were supported in parts by the National Science Foundation (NSF) under Contracts CCF 04-30654 and Project Emmitt of University of Texas at Dallas.

$\dagger$ The work of this author was supported in part by the NSF, Grant number CNS-0435297. effects of the different models on the pricing, the equilibrium threshold, the throughput, the revenue and the multiuser diversity gain.

This paper is organized as follows. In Section 2, a game-theoretic formulation is proposed. The analysis of the Nash equilibrium is presented in Section 3. Simulation results are discussed in Section 4 and conclusions are presented in Section 5.

\section{GAME MODEL}

In this section, we present a game-theoretic model for a distributed slotted system. We assume that all users can estimate their own channel characteristics by listening to a periodically-transmitted beacon signal from the base station (see also [4]).

Each slot of the system is a one-stage game. At the beginning of each slot, all users learn the current state of the game-- the number of users $(N)$ who currently have packets to send (active users) and their own channel characteristics. The number of active users can be estimated using the Pseudo-Bayesian Aloha algorithm in [5]. In this paper, we investigate the scenario where all the users are active users. Each of these users has two possible actions: transmit $(\mathrm{T})$ or wait (W). When a user transmits, its transmission can either succeed (S) or fail (F). The gain associated with a successful transmission is a normalized throughput of 1 , while the cost of transmission for user $i$ is $c_{i}$, and the network's current charge for the successful packet of user $i$ is $\mu_{i}$. If user $i$ transmits and succeeds in a given slot, then that user will receive a utility of $1-c_{i}-\mu_{i}$ for that slot (throughput cost - price paid). If the user refrains from transmission in a particular slot (waits), this will result in one slot delay for that user. In [3], we followed an aggressive approach toward transmission and thus this delay is associated with the loss in the throughput the user could have achieved if it transmitted and succeeded, which is determined as $-\left(1-c_{i}-\mu_{i}\right)$. However, for some services, the delay cost may be constant, e.g., for a delay-insensitive user, the delay cost may be zero. Thus, in this paper, we propose a generalized utility function for the waiting cost, i.e., $v\left(c_{i}, \mu_{i}, b\right)$, which is a function of $c_{i}, \mu_{i}$ and a constant delay cost $b$. If user $i$ transmits but fails, it will incur a transmission $\operatorname{cost} c_{i}$ as well as a one-slot delay, but it will not pay for the transmission (we assume that a user is charged only for successful transmissions). Therefore, the utility of this user in this case can be defined as $\left.-v\left(c_{i}, \mu_{i}, b\right)\right)-c_{i}$. Each user's goal is to maximize its own utility. The utility function for user $i$ is summarized as

$$
\text { Utility }=\left\{\begin{array}{cc}
1-c_{i}-\mu_{i} & \mathrm{~T} \text { and } \mathrm{S} \\
-v\left(c_{i}, \mu_{i}, b\right) & \mathrm{W} \\
-c_{i}-v\left(c_{i}, \mu_{i}, b\right) & \mathrm{T} \text { but } \mathrm{F}
\end{array} .\right.
$$


It is important to note that the transmission cost $c_{i}$ should be a normalized function in the range of $(0,1)$ and is related to the transmission energy cost. In wireless systems, channels change from one slot to another. The higher the channel power gain, the smaller the transmission power (hence transmission cost) to achieve the same spectral efficiency. Thus, the transmission cost should be defined as a monotonically-decreasing function of the channel power gains.

\section{EQUILIBRIA FOR THE COLLISION MODEL}

In this paper, we consider the classical collision model, where a transmission is considered to be successful only when a single user transmits. We consider a fair game model, where all users access the system with the same probability $p$. Then, the utility user $i$ gets from selecting action $\mathrm{T}$ is

$U_{i}(T)=\left(-c_{i}-v\left(c_{i}, \mu_{i}, b\right)\right)\left[1-(1-p)^{N-1}\right]+\left(1-c_{i}-\mu_{i}\right)(1-p)^{N-1}$

The utility user $i$ gets with action $\mathrm{W}$ is

$$
U_{i}(W)=-v\left(c_{i}, \mu_{i}, b\right) .
$$

In this paper, our goal is to optimize the system performance by appropriately selecting the price $\mu_{i}$ according to the transmission costs $\left\{c_{i}, i=1, \cdots, N\right\}$ of the users, so that each user can achieve its maximum utility by taking appropriate actions: $\mathrm{T}$ or $\mathrm{W}$. If the utility of $\mathrm{T}$ is larger than $\mathrm{W}$, then the user transmits. Otherwise, he waits. Then, the Nash equilibrium can be defined as follows:

A Nash equilibrium strategy will be a profile of strategies $\left(s_{1}^{*}, \ldots, s_{N}^{*}\right)$, where $s_{i}^{*} \in\{T, W\}$, such that for player $i, \forall s_{i} \in$ $\{T, W\}, U_{i}\left(s_{i}^{*}, s_{-i}^{*}\right) \geq U_{i}\left(s_{i}, s_{-i}^{*}\right)$, where $s_{-i}=s_{j \neq i}$.

Note that $U_{i}($.$) is defined in (2) and (3) and the effects of the$ other players are reflected by $p$. This equilibrium changes from one slot to another as the transmission costs change. To maximize its own utility, a user will take an action $T$ when $U_{i}(T) \geq U_{i}(W)$. Otherwise, it will wait. From (2) and (3), we can obtain

$$
U_{i}(T) \geq U_{i}(W) \Rightarrow c_{i} \leq\left(1+v\left(c_{i}, \mu_{i}, b\right)-\mu_{i}\right)(1-p)^{N-1} .
$$

From (4), we can obtain

$$
c_{i} \leq f_{i}\left(\mu_{i}, b, p\right)
$$

where $f_{i}$ is a function of $\mu_{i}, b$ and $p$.

Define

$$
\tau_{i}=f_{i}\left(\mu_{i}, b, p\right),
$$

where $\tau_{i}$ is called the equilibrium threshold. Then we have

$$
c_{i} \leq \tau_{i}
$$

Note that each user estimates his channel power gain through a periodically transmitted beacon signal and learns the best strategy periodically by comparing the transmission cost $c_{i}$ (which is a function of channel power gain) with the equilibrium threshold $\tau_{i}$. Once $\tau_{i}$ is obtained, each user can decide whether it transmits or not. Then, the problem of finding a Nash equilibrium strategy is reduced to finding an equilibrium threshold. The users whose transmission costs are smaller than the equilibrium threshold will transmit their packets at this time slot.

Now, let us analyze the relationship among $\tau_{i}, p$ and $\mu_{i}$. Since $p$ is the probability that a user transmits, we have

$$
p=P\left\{U_{i}(T) \geq U_{i}(W)\right\}=P\left\{c_{i} \leq \tau_{i}\right\} \triangleq F_{c_{i}}\left(\tau_{i}\right),
$$

where $F_{c_{i}}$ denotes the cumulative probability distribution function (CDF) of $c_{i}$. We obtain

$$
\tau_{i}=F_{c_{i}}^{-1}(p)
$$

and

$$
\mu_{i}=f_{i}^{-1}\left(\tau_{i}, b, p\right)=f_{i}^{-1}\left(F_{c_{i}}^{-1}(p), b, p\right),
$$

from (8) and (6), respectively. It can be seen from (9) that the equilibrium threshold $\tau_{i}$ does not change with the different utility functions.

Examples:

1. Aggressive model in [3]: $v_{i}=\left(1-c_{i}-\mu_{i}\right)$, and $f_{i}\left(\mu_{i}, b, p\right)=$ $\frac{2\left(1-\mu_{i}\right)(1-p)^{N-1}}{1+(1-p)^{N-1}}$ and $\mu_{i}=1-\frac{\tau_{i}+\tau_{i}(1-p)^{N-1}}{2(1-p)^{N-1}}=1-$ $\frac{F_{c_{i}}^{-1}(p)+F_{c_{i}}^{-1}(p)(1-p)^{N-1}}{2(1-p)^{N-1}}$.

2. Constant model: $v_{i}=b$. The waiting cost is a constant, which is reasonable for the delay-insensitive services. Thus $f_{i}\left(\mu_{i}, b, p\right)=\left(1+b-\mu_{i}\right)(1-p)^{N-1}$ and $\mu_{i}=1+b-$ $\frac{\tau_{i}}{(1-p)^{N-1}}=1+b-\frac{F_{c_{i}}^{-1}(p)}{(1-p)^{N-1}}$.

\subsection{Throughput Optimization}

Here, we describe how the network adjusts pricing to achieve the maximum throughput. It is well known that the network throughput $S$ of a slotted Aloha system is

$$
S=N p(1-p)^{N-1}
$$

and the maximum throughput is achieved when $p=1 / N$ [5]. To obtain the same maximum throughput, we set the transmission probability to $p=1 / N$. Therefore, the equilibrium threshold is obtained from (9), i.e.,

$$
\tau_{i}=F_{c_{i}}^{-1}\left(\frac{1}{N}\right),
$$

and the optimal pricing strategy to maximize the throughput is obtained from (10), i.e.,

$$
\mu_{i}=f_{i}^{-1}\left(\tau_{i}, b, p\right)=f_{i}^{-1}\left(F_{c_{i}}^{-1}\left(\frac{1}{N}\right), b, \frac{1}{N}\right),
$$

\subsection{Revenue Optimization}

In the previous subsection, the network decides the price aiming to maximize the throughput of the whole network. In this subsection, we consider a more realistic objective for the service provider which is to maximize revenue. Assume that the network charges only for successful transmissions. To achieve fairness, the transmission probabilities for all users are enforced to be the same and hence the revenue for the network is given by

$$
R=\sum_{i=1}^{N} \mu_{i} p(1-p)^{N-1} .
$$

Substituting $\mu_{i}$ given in (10) into (14) and setting the derivative of (14) to zero, we can obtain the optimum $p$, and further obtain $\tau_{i}$ and $\mu_{i}$ from (9) and (10), respectively.

\section{SIMULATION RESULTS}

The simulation results are obtained from 100, 000 independent frames and we assume a Bit-Error-Rate requirement of $\mathrm{BER}=10^{-5}$ and a corresponding Signal-to-Noise ratio requirement of $\mathrm{SNR}=15 \mathrm{~dB}$. 


\subsection{System Model}

We assume that the channels between users and the access point are characterized by independent fading. The lowpass equivalent channel gains $\left\{h_{i}, i=1,2, \ldots N\right\}$ between an arbitrary user $i$ and the access point are assumed to be independent zero-mean unit-variance circularly-symmetric complex Gaussian random variables. Consequently, the channel power gains $G_{i}=\left|h_{i}\right|^{2}(i=1,2, \ldots, N)$ have exponential distribution with mean ' 1 ', i.e,

$$
F_{i}\left(G_{i}\right)=\left(1-e^{-G_{i}}\right) u\left(G_{i}\right),
$$

where $u\left(G_{i}\right)$ is the unit-step function. We define the transmission cost to be $c_{i}=1-F_{i}\left(G_{i}\right)$. This definition preserves the required properties for $c_{i}$, namely, normalization, and monotonically decreasing with increased channel power gains ${ }^{2}$. Thus, we obtain

$$
F_{c_{i}}\left(\tau_{i}\right)=P\left\{1-F_{i}\left(G_{i}\right)<\tau_{i}\right\}=P\left\{G_{i}>F_{i}^{-1}\left(1-\tau_{i}\right)\right\}=\tau_{i} .
$$

From (9) and (10), we obtain

$$
\tau_{i}=p \triangleq \tau, \quad i=1,2, \ldots, N,
$$

and

$$
\mu_{i}=f_{i}^{-1}\left(\tau_{i}, b, p\right)=f_{i}^{-1}(p, b, p) .
$$

\section{Examples revisited:}

1. Aggressive model in [3]: The optimum thresholds and pricing are given in [3].

2. Constant model: (18) becomes

$$
\mu_{i}=1+b-\frac{p}{(1-p)^{N-1}} \triangleq \mu, \quad i=1,2, \ldots, N .
$$

For throughput optimization, the threshold and the pricing can be obtained from (12) and (19), respectively, as $\tau=\frac{1}{N}$ and $\mu=1+b-\frac{\frac{1}{N}}{\left(1-\frac{1}{N}\right)^{N-1}}$. For revenue optimization, $R$ becomes

$$
R=N\left(1+b-\frac{p}{(1-p)^{N-1}}\right) p(1-p)^{N-1} .
$$

The optimum $p$ can be obtained from (20). Then, the equilibrium threshold and pricing can be obtained from (17) and (19), respectively.

\subsection{Numerical and Simulation Results}

\subsubsection{Threshold and Pricing Comparison}

The pricing and threshold comparisons are given in Fig. 1 and 2, respectively. From Fig. 1, we can see that the pricing of the constant model at $b=0$ is slightly smaller than that of the aggressive model for a small number of users and is almost the same pricing for a large number of users. It can also be seen from Fig. 1 that the pricing for the constant model increases with the value of $b$, which can also be seen from (19). This is intuitively appealing since the users tend to transmit when the waiting cost is high and the network operator has to increase the price to discourage the users from transmission to reduce collisions.

From Fig. 2, we can see that the constant model has exactly the same threshold as the aggressive model when both are based on throughput optimization, which is $\frac{1}{N}$ and does not vary with $b$. For revenue

\footnotetext{
${ }^{2}$ Note that there may be some other definitions of $c_{i}$ satisfying the required properties.
}

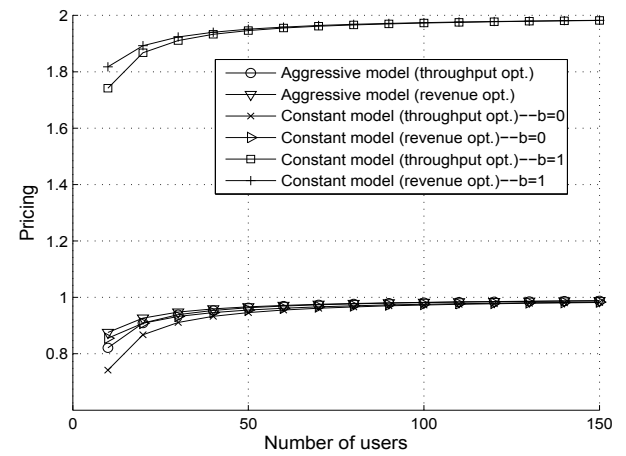

Fig. 1. Pricing comparison

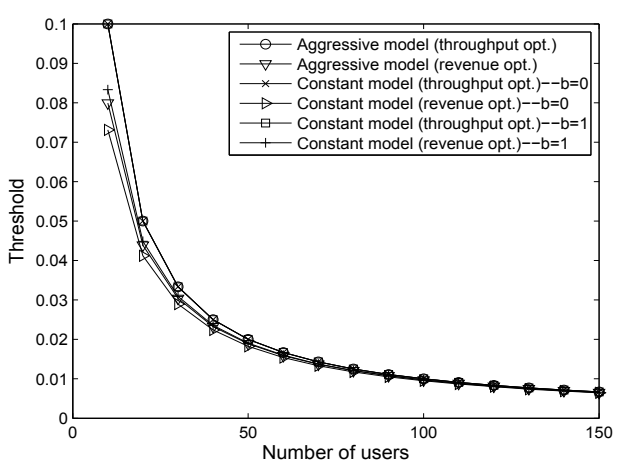

Fig. 2. Threshold comparison

optimization, the threshold of the constant model varies slightly with $b$ and is only slightly different from the aggressive model.

\subsubsection{Throughput and Revenue Comparison}

Fig. 3 compares the throughput of the aggressive model and the constant model. Under the throughput optimization framework, both models have exactly the same throughput. This is due to fact that the equilibrium threshold does not change with different models as seen from (12) and Fig. 2. However, under the revenue optimization framework, the constant model with $b=1$ achieves higher throughput than the aggressive model, but the latter gives a higher throughput than the former with $b=0$.

Fig. 4 compares the revenue of the aggressive model and the constant model. The aggressive model has almost the same revenue as constant model with $b=0$. However, the constant model with $b=1$ achieves much higher revenue than the aggressive model. The reason is that the pricing of the constant model with $b=1$ is much higher than that of the aggressive model and the constant model with $b=0$ (see Fig. 1).

\subsubsection{Spectral Efficiency and Energy Consumption Comparison}

To show the multiuser diversity gains, we will consider two performance criteria: spectral efficiency and energy consumption.

1. Spectral Efficiency: Here, a continuous-rate adaptive modulation is used by exploiting the instantaneous channel power gains, and the spectral efficiency is calculated as

$$
r=\log _{2}\left(1+\frac{\gamma P G_{i}}{\sigma_{i}^{2}}\right)
$$

where $P$ is the transmission power, $\sigma_{i}^{2}$ is the noise power and $\gamma$ is the SNR gap given by $\gamma=-\frac{1.5}{\ln (5 \mathrm{BER})}$. Note that 




Fig. 3. Throughput comparison

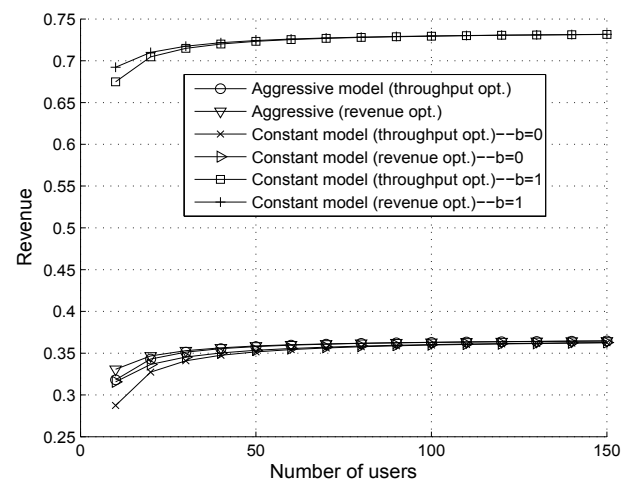

Fig. 4. Revenue comparison

to show the effects of the channel gains on the number of bits transmitted, $P$ is considered fixed for all the users and is set to be ' 1 ' in the simulation.

2. Energy Consumption: From (21), we can calculate the power $P$ to achieve data rate $r$ as follows:

$$
P=\frac{\left(2^{r}-1\right) \sigma_{i}^{2}}{\gamma G_{i}}
$$

In the simulation, we use the normalized data rate $r=1$.

It can be seen from Figs. 5 and 6 that the constant model and the aggressive model based on the throughput optimization have exactly the same spectral efficiency and energy consumption since they have the same equilibrium thresholds as shown in Fig. 2. Under the revenue optimization framework, the spectral efficiencies achieved by different models are almost the same. However, energy consumption varies slightly with $b$. The energy consumption of the constant model at $b=0$ is less than that of the aggressive model, which is, in turn, less than that of the constant model at $b=1$. This is due to the fact that the threshold (or transmission probability) of the constant model at $b=0$ is smaller than that of the aggressive model, which is less than that of the constant model at $b=1$ (see Fig. 2).

Another observation from Figs. 5 and 6 is that the channel aware Aloha given in [4] (which is well known as a distributed scheme achieving multiuser diversity gain) achieves almost the same spectral efficiency as both constant model and aggressive model while it consumes more energy than them based on the revenue optimization framework. Note that spectral efficiency and energy consumption are related to each other through (21) and (22), but the effect of multiuser diversity is more pronounced in the energy consumption.

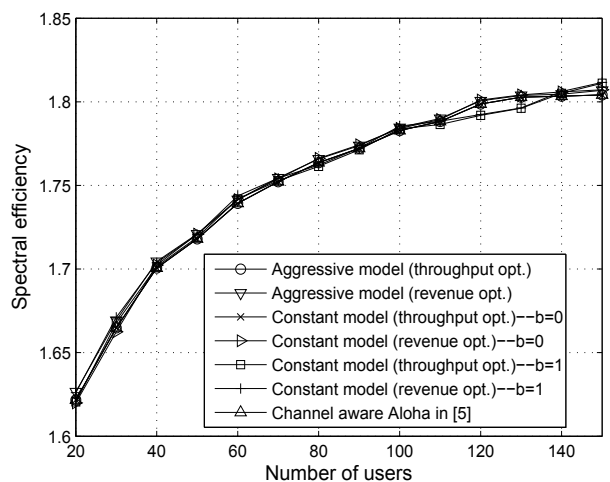

Fio 5 Snectral efficiency comnaricon



Fig. 6. Energy comparison

\section{CONCLUSION}

In this paper, a generalized utility function for the waiting cost is proposed. We have shown that different models have no impact on the achieved throughput, spectral efficiency and energy consumption under the throughput optimization while they do have slight effects under the revenue optimization. Compared to the channel aware Aloha in [4], our generalized utility function model achieves higher multiuser diversity gain by deploying the pricing strategy even if all the users act selfishly, and demonstrates flexibility in designing utility functions associated with different QoS requirements, in order to optimize the overall network performance.

\section{REFERENCES}

[1] R. Knopp and P. A. Humblet, "Information capacity and power control in single-cell multiuser communications," in Proc. IEEE Int. Conf. Commun, Seattle, WA, Jun. 1995, pp. 331-335.

[2] E. Altman, D. Barman, R. El Azouzi, and T. Jimenez, "A game theoretic approach for delay minimization in slotted aloha," in Proc. IEEE Int. Conf. Commun., Paris, Jun 2004, vol. 7, pp. 3999-4003.

[3] C. Comaniciu, D. Wang, H. Minn, and N. Al-Dhahir, "A game theoretic solution for exploiting multiuser diversity in cooperative slotted aloha," in IEEE Int. Conf. Commn., 2007.

[4] X. Qin and R. Berry, "Exploiting multiuser diversity for medium access in wireless networks," in Proc. of IEEE INFOCOM Conf., San Francisco, CA, Apr. 2003, vol. 2, pp. 1084-1094.

[5] D. Bertsekas and R. Gallager, Data Networks, Prentice-Hall, Upper Saddle River, NJ, 2nd edition, 1992. 